shape soil structure (Sci. Rep. 8, 10236, 2018). Increasingly popular with farmers are biological sensors because they allow near-real-time detection of airborne pathogen spores. Crop losses due to pathogens amount to billions of dollars annually. Such systems allow early intervention, helping farms avoid the cost and environmental impact of field-wide chemical pesticide treatments.

An early warning system for pathogens developed by researchers at Rothamsted Research, an agricultural research center in Harpenden, UK, samples the air every evening and alerts growers of a pathogen's arrival via a mobile phone network. The system detects and identifies spores by loop-mediated isothermal amplification (LAMP) assays (Nucleic Acids Res. 15, E63, 2000), says Jon West, a plant pathologist and aerobiologist at Rothamsted. LAMP assays detect target genes using a single container at a single temperature and so are faster than the more widely used polymerase chain reaction method. The pathogen detection system can identify potato late blight, the Sclerotinia fungus and several sugar beet pathogens, he says. Allele-specific LAMP assays could be used to detect fungicide resistance alleles, says West. "That means we can not only make a detection of the pathogen before it infects, but we can advise farmers if that pathogen is resistant to a particular fungicide," he says.

Farmers also need to be able to detect insects that damage crops. FaunaPhotonics, a startup in Copenhagen, is developing an insect-detection system based on lidar, a type of radar that uses laser light instead of radio waves. The system collects data on insect body mass and wing beat frequency, and uses deep learning algorithms to classify insect species flying over fields. The technology is a higher-resolution version of an insect-detecting vertical radar system developed at Rothamsted in the early 2000s.

To spot and identify diseases, or to check for ripeness, growers and agronomists have been processing vast quantities of images-including those generated from computer vision systems in fields-using machine learning. There has also been a proliferation of companies using machine learning for plant breeding (Nat. Biotechnol. 35, 397-398, 2017). But researchers and companies are just beginning to develop machine learning systems that attempt to model whole agronomic systems.

Yoshua Bengio, a computer scientist and pioneer of deep learning at the University of Montreal, says it will take some time before agronomists will be able to collect all agriculturally relevant variables needed to train machine learning systems. Only then will the systems accurately predict outcomes of treatments and other changes, he says. "The data collection isn't there yet," he says.

Companies developing machine learning for agriculture first need to ensure the data are collected in sufficient quantities and from sufficiently diverse places, says Bengio. It is likely that datasets will expand over the next few years given the growing adoption in agriculture of drones and sensors, and image analysis is likely to be the first area where machine learning will become widespread. "We have tons of data from satellites," he says. "They need to be labeled, but it's not as difficult as having to create the measurements in the first place."

The models used in machine learning may also need adapting. Agriculture is a highly complex system with large numbers of variables and high degrees of uncertainty, and under these conditions machine learning systems struggle to make useful predictions. An alternative approach is to build probabilistic models based on Bayesian networks, which can reason well even with incomplete information and readily update models with new information.

This is the strategy taken by SALUS, a program that captures the interactions taking place in soil-plant-atmosphere systems. SALUS (System Approach to Land Use Sustainability) was developed by Bruno Basso, an agricultural systems scientist at Michigan State University. "SALUS accounts for the effects of soil type, climate, genotype and agronomic management on crop yield, grain quality and environmental outcomes," he says.
SALUS simulations are dynamic and can model the agricultural systems of whole countries. Basso and colleagues have used it in projects funded by the Food and Agriculture Organization of the United Nations, the World Bank, the European Union, the US Agency for International Development, the US Department of Agriculture, and The Bill and Melinda Gates Foundation, among others, according to Basso.

The SALUS system has been scaled up and commercialized by $\mathrm{CiBO}$ Technologies of Cambridge, Massachusetts. It is not meant to help individual farmers manage their operations. Rather, it's designed to allow international organizations, national governments and multinational corporations to run what-if scenarios about climate change, agriculture policy and product development. "It's essentially this powerful inference engine," says David Worn, CiBO's CEO.

The system can aid decision-making during field trials for agricultural product development, says Worn. "We bring a lot of that process in silico," he says. This cuts about $30 \%$ of field trial costs and one to three years from the product development lifecycle, according to Worn.

CiBO's system recreates a plant's responses to its environment in silico. The system incorporates a wide breadth of data into the model, from satellite images, farm management information and climate data to vast amounts of scientific data about plant and soil biology. "It's really a physical simulation of the coupled plant-soilatmosphere-management ecosystem," says Ryan Richt, CiBO's vice president of engineering.

The high resolution of the model allows the system to infer more information from, say, a leaf-reflectance index, than other systems, and the system can, in turn, use that data to improve the model. "A plant is an integrated sensor of its environment," says Richt. "So if you can reverse engineer what it's telling you, you can know with much more spatial resolution and precision what's going on than having some small number of physical sensors distributed out in the field."

Eric Smalley Boston

\title{
PODCAST
}

\section{First rounders: Feng Zhang}

Feng Zhang runs the Zhang Lab at the Massachusetts Institute of Technology. He's also a faculty member at the Broad Institute of MIT and Harvard, and has co-founded several biotech companies, including Editas Medicine. His conversation with Nature Biotechnology covers immigrating to America as a boy, his moment of discovery with CRISPR, and what massive success before the age of 35 does to a researcher.

http://www.nature.com/nbt/podcast/index.html

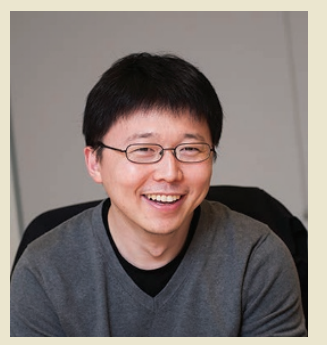

